\title{
Conhecimento sobre o tratamento precoce e as medidas de prevenção da Covid-19 em moradores do Estado do Pará-Brasil
}

Knowledge about early treatment and prevention measures for Covid-19 in residents of the State of Pará-Brazil

Conocimiento sobre medidas de prevención y tratamiento temprano de Covid-19 en residentes del Estado de Pará-Brasil

\section{Resumo}

Desde o início da pandemia, vários fármacos, foram estudados como alternativas para tratar a infecção pela COVID19. Entre estes, pode-se citar a cloroquina, a ivermectina, a hidroxicloroquina e a nitazoxanida. O presente estudo buscou identificar o conhecimento e comportamento sobre o uso de medicamentos sem comprovação científica em parte da população do estado do Pará, durante a pandemia de COVID-19. A pesquisa adotou uma abordagem descritiva e prospectiva, envolvendo um questionário em formulário eletrônico, para coletar dados de moradores do estado. Os resultados demonstraram que a frequência de indivíduos que acreditavam no tratamento precoce e/ou utilização de medicamentos como profilaxia foi maior em residentes das cidades do interior do estado e nas faixas etárias maiores, apesar dos achados científicos contrários. Na população estudada, 9,9\% dos residentes das demais cidades não acreditavam que as vacinas eram seguras e eficientes e 7,0\% relataram que apenas algumas seriam. A orientação ideológica de uma parte da população e os movimentos antivacinas, podem explicar em parte os resultados apresentados. O uso inadequado de medicamentos para o tratamento precoce e/ou de uso profilático da COVID-19 ainda é uma realidade no estado Pará, devendo serem implementadas políticas públicas para coibir tal uso e incremento de ações que busquem conscientizar sobre a importância da vacinação e manutenção das medidas de prevenção cientificamente comprovadas.

Palavras-chave: Covid-19; Epidemiologia; Saúde pública; Tratamento medicamentoso.

\section{Abstract}

Since the beginning of the pandemic, several drugs have been studied as alternatives to treat COVID-19 infection. These include chloroquine, ivermectin, hydroxychloroquine and nitazoxanide. The present study sought to identify the 
knowledge and behavior about the use of drugs without scientific evidence in part of the population of the state of Pará, during the COVID-19 pandemic. The research adopted a descriptive and prospective approach, involving a questionnaire in an electronic form, to collect data from residents of the state. The results showed that the frequency of individuals who believed in early treatment and/or use of medication as prophylaxis was higher in residents of cities in the interior of the state and in older age groups, despite the contrary scientific findings. In the population studied, $9.9 \%$ of residents in other cities did not believe that vaccines were safe and effective, and $7.0 \%$ reported that only a few would be. The ideological orientation of a part of the population and anti-vaccination movements can partly explain the results presented. The inappropriate use of drugs for the early treatment and/or prophylactic use of COVID-19 is still a reality in the state of Pará, and public policies must be implemented to curb such use and increase actions that seek to raise awareness about the importance of vaccination and maintenance of scientifically proven prevention measures.

Keywords: Covid-19; Epidemiology; Public health; Drug treatment.

\section{Resumen}

Desde el comienzo de la pandemia, se han estudiado varios fármacos como alternativas para tratar la infección por COVID-19. Estos incluyen cloroquina, ivermectina, hidroxicloroquina y nitazoxanida. El presente estudio buscó identificar el conocimiento y comportamiento sobre el uso de drogas sin evidencia científica en parte de la población del estado de Pará, durante la pandemia COVID-19. La investigación adoptó un enfoque descriptivo y prospectivo, que incluyó un cuestionario en formato electrónico, para recopilar datos de los residentes del estado. Los resultados mostraron que la frecuencia de personas que creían en el tratamiento temprano y / o el uso de medicamentos como profilaxis fue mayor en los habitantes de las ciudades del interior del estado y en los grupos de mayor edad, a pesar de los hallazgos científicos contrarios. En la población estudiada, el 9,9\% de los residentes en otras ciudades no creían que las vacunas fueran seguras y efectivas, y el 7,0\% informó que solo unas pocas lo serían. La orientación ideológica de una parte de la población y los movimientos antivacunas pueden explicar en parte los resultados presentados. El uso inadecuado de medicamentos para el tratamiento temprano y / o uso profiláctico del COVID-19 es todavía una realidad en el estado de Pará, y se deben implementar políticas públicas para frenar dicho uso e incrementar las acciones que busquen concientizar sobre la importancia de la vacunación y mantenimiento de medidas de prevención científicamente probadas.

Palabras clave: Covid-19; Epidemiología; Salud pública; Tratamiento farmacológico.

\section{Introdução}

A COVID-19 é uma doença respiratória aguda que foi descoberta no final de 2019, com um forte impacto mundial. Evidências epidemiológicas indicam que esse novo coronavírus humano (SARS-CoV-2) se originou em morcegos e se espalhou através de outros animais nos mercados de Guangdong na China, infectando a população humana a partir do manuseio ou consumo dessas espécies exóticas (Ictv, 2020). Em dezembro de 2019, várias unidades locais de saúde relataram grupos de pacientes com pneumonia de causa desconhecida que estavam epidemiologicamente ligados à um mercado de frutos do mar e animais aquáticos em Wuhan, província de Hubei, China (Zhu et al., 2020).

A disseminação do SARS-CoV-2 foi exponencial, atingindo rapidamente cerca de 19 países, com transmissão autóctone na China, Alemanha, Japão, Vietnã e Estados Unidos da América. Nesse sentido, em 30 de janeiro de 2020 , a Organização Mundial de Saúde (OMS), através do Comitê de Emergência do Regulamento Sanitário Internacional, declarou que o surto do novo coronavírus constituía uma Emergência de Saúde Pública de Importância Internacional (Who, 2020).

De acordo com a OMS, desde o início da pandemia, ocorreram 216.867.420 de casos confirmados e 4.507.837 de óbitos até o dia 31 de agosto de 2021. No Brasil, é declarada Emergência em Saúde Pública de Importância Nacional em 03 de fevereiro de 2020, sendo que o primeiro caso de infecção por SARS-CoV-2, foi confirmado em 26 de fevereiro e em 3 de março, a segunda confirmação, sem evidência de transmissão local. Os dois primeiros casos eram de indivíduos do sexo masculino, residentes na cidade de São Paulo, que haviam regressado de viagem à Itália (Brasil, 2020a). Até 31 de agosto de 2021, o Brasil possuía 20.741.815 casos, sendo o segundo país do mundo em número de mortes pela COVID-19 (Who, 2021). O estado do Pará está situado na região norte do Brasil, na chamada Amazônia Legal, e teve o primeiro caso confirmado de COVID-19 em 18 de março de 2020. Até o dia 31 de agosto de 2021, o estado possuía 584.363 de casos confirmados e 16.461 óbitos (Sespa, 2021a). 
Vários fármacos foram estudados como alternativas para tratar a infecção pela COVID-19. Isto seria feito a partir da técnica de reposicionamento de medicamentos. Entre estes, pode-se citar a cloroquina, a ivermectina, a hidroxicloroquina e a nitazoxanida. Todavia, até a data de 29/03/2021, vários testes clínicos já haviam sido realizados, que não comprovaram a eficácia de tais medicamentos contra a COVID-19 (Santos-Pinto et al., 2021). Por isso, vários países desaconselham a utilização desses medicamentos na COVID-19 (Cdc, 2020). Apesar disso, o governo federal do Brasil optou por continuar a incentivar o uso, principalmente da cloroquina e hidroxicloroquina. Como mostra alguns protocolos de manejo, de pacientes com COVID-19 (Brasil, 2020b).

Sendo assim, os indivíduos procuraram na internet, maneiras de se protegerem do contágio do citado vírus, ficando expostas a mensagens com conteúdo falso, sobre as medidas de prevenção e tratamento precoce (Ses-MT, 2020).O estado do Pará carece de informações sobre a utilização de medicamentos sem comprovação científica para o tratamento e/ou como medida profilática da COVID-19. O presente estudo buscou identificar o conhecimento e comportamento sobre o uso de medicamentos sem comprovação científica em parte da população do estado do Pará, durante a pandemia de COVID-19.

\section{Metodologia}

O estudo ocorreu entre os dias 03 de julho e 31 de agosto de 2021 e adotou uma abordagem descritiva e prospectiva, envolvendo um questionário autoadministrado, por formulário eletrônico, para coletar dados pessoais de moradores do estado do Pará. Com relação aos aspectos éticos, este foi submetido à Plataforma Brasil sob CAAE n 48369821.3.0000.0018 e aprovado via Parecer Consubstanciado n 4.824.889. Os critérios de inclusão foram pessoas maiores de 18 anos; de ambos os sexos; moradoras do estado do Pará; que aceitaram participar do estudo, autorizando com o Termo de Consentimento Livre Esclarecido (TCLE) online e os de exclusão, foram pessoas menores de 18 anos; moradoras de outros estados; que não aceitaram participar do estudo ou não autorizaram o mesmo.

O questionário foi desenvolvido na plataforma Google Forms ${ }^{\circledR}$, contendo questões fechadas (de múltipla escolha). A participação na pesquisa foi voluntária e consentida, através do convite eletrônico contendo o link de acesso ao formulário, encaminhado por meio digital (e-mail e/ou aplicativos de comunicação social). Os dados foram exportados da Plataforma Google Forms® para o programa Microsoft Excel $365 \circledR$, onde foram confeccionadas planilhas. Para análise estatística, foi utilizado o programa BioEstat ${ }^{\circledR} 5.3$ e adotado o nível de significância de $5 \%$.

\section{Resultados e Discussão}

Foram incluídos no estudo 242 indivíduos, que se enquadravam aos critérios de inclusão. As características socioeconômicas, estão descritas na Tabela 1. 
Tabela 1: Perfil socioeconômico dos participantes do estudo.

\begin{tabular}{|c|c|c|}
\hline Variável analisada & Indivíduos (n) & $\%$ \\
\hline \multicolumn{3}{|l|}{ Sexo } \\
\hline Masculino & 77 & $31,8 \%$ \\
\hline Feminino & 165 & $68,2 \%$ \\
\hline \multicolumn{3}{|l|}{ Local de residência } \\
\hline Belém & 171 & 70,6 \\
\hline Demais cidades & 71 & 29,4 \\
\hline \multicolumn{3}{|l|}{ Faixa etária } \\
\hline $18-39$ & 168 & $69,4 \%$ \\
\hline $40-60$ & 64 & $26,4 \%$ \\
\hline Maior que 60 anos & 10 & $4,2 \%$ \\
\hline \multicolumn{3}{|l|}{ Grau de instrução } \\
\hline Fundamental (incompleto + completo) & 3 & $1,2 \%$ \\
\hline Médio (incompleto + completo) & 37 & $15,3 \%$ \\
\hline Superior (incompleto + completo) & 202 & $83,5 \%$ \\
\hline \multicolumn{3}{|l|}{ Situação marital } \\
\hline Casado(a) ou em união estável & 92 & $38,0 \%$ \\
\hline Divorciado/Separado (a) & 9 & $3,8 \%$ \\
\hline Solteiro (a) & 138 & $57,0 \%$ \\
\hline Viúvo (a) & 3 & $1,2 \%$ \\
\hline \multicolumn{3}{|l|}{ Renda familiar mensal } \\
\hline Sem rendimento & 17 & $7,0 \%$ \\
\hline Entre 1 e 2 salários-mínimos & 92 & $38,0 \%$ \\
\hline Entre 3 e 4 salários-mínimos & 75 & $31,0 \%$ \\
\hline Mais de 4 salários-mínimos & 58 & $24,0 \%$ \\
\hline
\end{tabular}

Fonte: Silva et al. (2021).

Quanto ao conhecimento acerca do tratamento precoce, observou-se que 22,0\% dos homens e $17,6 \%$ das mulheres; 15,2\% dos residentes de Belém e 28,2\% dos residentes das demais cidades; $11,9 \%$ da faixa etária de 18 - 39 anos, $37,5 \%$ dos de 40 - 60 anos e $20 \%$ dos maiores de 60 anos, acreditavam que o tratamento precoce com medicamentos como hidroxicloroquina, cloroquina, ivermectina e nitazoxanida poderiam curar a COVID-19 ou evitar que o paciente desenvolvesse as formas mais graves da doença (Tabela 2). Houve diferença estatisticamente significante quanto ao local de residência ( $p$ : $0,0307)$ e a idade $(p: 0,0002)$. 
Tabela 2: Indivíduos que acreditavam no tratamento precoce da COVID-19.

\begin{tabular}{cccccc}
\hline Variável & Sim & $\%$ & Não & $\%$ & $p$ \\
\hline Sexo & 17 & 22,0 & 60 & 78,0 & \\
Masculino & 29 & 17,6 & 136 & 82,4 & 0,5121 \\
Feminino & 26 & 15,2 & 145 & 84,8 & \\
Local de residência & 20 & 28,2 & 51 & 71,2 & 0,0307 \\
Belém & & & & & \\
Demais cidades & 20 & 11,9 & 148 & 88,1 & \\
Idade & 24 & 37,5 & 40 & 62,5 & \\
$18-39$ & 2 & 20,0 & 8 & 80,0 & 0,0002 \\
$40-60$ & & & & & \\
Maior que 60 & & &
\end{tabular}

Fonte: Silva et al. (2021)

Quanto a utilização de medicamentos como forma profilática da COVID-19, 16,9\% dos homens e 8,4\% das mulheres; $8,8 \%$ dos residentes de Belém e 16,9\% dos residentes das demais cidades; 5,3\% da faixa etária de 18 - 39 anos, $25 \%$ dos de 40 - 60 anos e 20\% dos maiores de 60 anos, acreditavam na efetividade de tais medicamentos (Tabela 3). Houve diferença estatisticamente significante quanto a idade dos participantes ( $p: 0,0004)$.

Tabela 3: Indivíduos que acreditavam em medicamentos como forma profilática.

\begin{tabular}{cccccc}
\hline Variável & Sim & $\%$ & Não & $\%$ & $p$ \\
\hline Sexo & & & & & \\
Masculino & 13 & 16,9 & 64 & 83,1 & 0,0866 \\
Feminino & 14 & 8,4 & 151 & 91,6 & \\
Local de residência & & & & & \\
Belém & 15 & 8,8 & 156 & 91,2 & \\
Demais cidades & 12 & 16,9 & 59 & 83,1 & \\
Idade & 9 & 5,3 & 159 & 94,7 & 75,0 \\
18-39 & 16 & 25,0 & 48 & 80,0 & 0,0004 \\
40-60 & 2 & 20,0 & 8 & & \\
Maior que 60 & & & & & \\
\hline
\end{tabular}

Fonte: Silva et al. (2021).

Entre os medicamentos utilizados nos protocolos de "tratamento precoce", destacam-se hidroxicloroquina, cloroquina, ivermectina e nitazoxanida. A cloroquina e a hidroxicloroquina, são medicamentos utilizados em infecções parasitárias, em doenças autoimunes e possuem atividade antiviral em estudos in vitro (Yan et al., 2013). Em março de 2020, um estudo publicado por Gautret et al. (2020), sugeria uma melhora clínica dos pacientes com COVID-19 após a utilização da hidroxicloroquina. Ainda em 2020, o Food and Drug Administration (FDA) e posteriormente a Organização Mundial da Saúde (OMS) em 2021, desaconselharam a utilização deles, após estudos multicêntricos concluírem acerca da ausência de eficácia da cloroquina/hidroxicloroquina no tratamento da COVID-19 (Recovery, 2020; Who, 2021).

A eficiência da hidroxicloroquina como medicamento profilático ou para utilização precoce, antes do aparecimento de sintomas para COVID-19, não foi comprovada (Boulware et al., 2020; Mitjá et al., 2021). Alguns estudos mostram que o uso indiscriminado destes medicamentos, está associado ao incremento da mortalidade dos pacientes, por conta dos efeitos adversos presentes (Singh et al. 2021; Axfors et al. 2021).

Quanto a ivermectina, um estudo de Caly et al. (2020) demonstrou in vitro a redução da replicação viral do SARSCoV-2 após 48 horas. Todavia, a dose necessária in vivo seria demasiadamente elevada, com risco de neurotoxicidade 
provocada pelo fármaco (Chaccour et al., 2020). Um estudo randomizado de López-Medina et al. (2021), com 476 pacientes com COVID-19 leve e em estágio inicial, não demonstrou diferença significativa no tempo de resolução de sintomas entre os grupos que receberam a ivermectina e o grupo controle, nem tampouco diferença na evolução clínica da doença.

A nitazoxanida é um antiparasitário de amplo espectro, possuindo ação antiviral in vitro contra o SARS-CoV-2. Todavia, um estudo publicado em 2020 não demonstrou diferença significativa no tempo de resolução de sintomas entre os grupos que receberam o fármaco em comparação aos que receberam placebo (Rocco et al., 2020).

Apesar de estudos comprovarem a ineficiência de tais fármacos no tratamento precoce da COVID-19, o Ministério da Saúde do Brasil divulga em maio de 2020, o informativo "Orientações para o manuseio medicamentoso precoce de pacientes com diagnóstico da COVID-19” (Brasil, 2020a).

Os resultados do presente estudo demonstraram que a frequência de indivíduos que acreditavam no tratamento precoce e/ou utilização de medicamentos como profilaxia foi maior em residentes das cidades do interior do estado e nas faixas etárias maiores, apesar dos achados científicos contrários. Um estudo publicado por Lima em 2021, sugere uma elevada orientação ideológica no governo federal do Brasil e em parte da população, interferindo diretamente na formulação de políticas públicas de saúde e segurança sanitária para a prevenção e combate da pandemia. Tal orientação ideológica pode explicar em parte os resultados apresentados.

Na população estudada, $91,0 \%$ dos e homens e das mulheres; $94,2 \%$ dos moradores de Belém e $83,1 \%$ dos residentes das demais cidades; $93,4 \%$ da faixa etária de 18 - 39 anos, $87,5 \%$ dos de $40-60$ anos e 70\% dos maiores de 60 anos, acreditavam que as vacinas utilizadas eram seguras e evitavam que a pessoa fosse contaminada, ou caso fosse, que não apresentassem as formas mais graves da doença (tabela 4). A diferença observada no local de residência, foi estatisticamente significante ( $p: 0,0291)$.

Tabela 4: Indivíduos que acreditavam que as vacinas são seguras e eficientes.

\begin{tabular}{|c|c|c|c|c|c|c|c|}
\hline Variável & Sim & $\%$ & Não & $\%$ & $\begin{array}{c}\text { Apenas } \\
\text { algumas } \\
\text { vacinas } \\
\text { funcionam } \\
\end{array}$ & $\%$ & $p$ \\
\hline \multicolumn{8}{|l|}{ Sexo } \\
\hline Masculino & 70 & 91,0 & 3 & 3,9 & 4 & 5,1 & \\
\hline Feminino & 150 & 91,0 & 8 & 4,8 & 7 & 4,2 & 0,9052 \\
\hline \multicolumn{8}{|l|}{ Local de residência } \\
\hline Belém & 161 & 94,2 & 4 & 2,3 & 6 & 3,5 & \\
\hline Demais cidades & 59 & 83,1 & 7 & 9,9 & 5 & 7,0 & 0,0291 \\
\hline \multicolumn{8}{|l|}{ Idade } \\
\hline $18-39$ & 157 & 93,4 & 6 & 3,6 & 5 & 3,0 & \\
\hline $40-60$ & 56 & 87,5 & 3 & 4,7 & 5 & 7,8 & \\
\hline Maior que 60 & 7 & 70,0 & 2 & 20,0 & 1 & 10,0 & 0,2388 \\
\hline
\end{tabular}

Fonte: Silva (2021).

Até o dia 11 de setembro de 2021, o estado do Pará havia administrado 6.967.343 doses de vacinas contra a COVID19 , apresentando uma cobertura de $51,19 \%$ da população vacinada com a primeira dose e $32,24 \%$ com a segunda dose. Os dados epidemiológicos mostravam uma redução de óbitos e casos confirmados a partir da semana epidemiológica 23 de 2021 (Sespa, 2021a, 2021b). Na população estudada, 9,9\% dos residentes das demais cidades não acreditavam que as vacinas eram seguras e eficientes e 7,0\% relataram que apenas algumas seriam. Beltrão et al. (2020) sugerem que os movimentos antivacinas vem ganhando força e um maior espaço nas mídias digitais. Isso se deve a crescente influência que a internet e as redes sociais 
exercem sobre a vida dos usuários. No presente estudo, 51,6\% dos participantes relataram utilizar as redes sociais para buscar informações sobre a COVID-19 e 18,2\% utilizavam aplicativos de trocas de mensagens, o que pode explicar em parte os resultados apresentados.

\section{Considerações Finais}

Sugere-se que o uso inadequado de medicamentos para o tratamento precoce e/ou de uso profilático da COVID-19 ainda é uma realidade no estado do Pará. Todavia, o conhecimento científico atual não justifica a utilização destes, devendo serem implementadas políticas públicas para coibir tal uso e incremento de ações que busquem conscientizar sobre a importância da vacinação e manutenção das medidas de prevenção cientificamente comprovadas. Novos estudos com um número maior de indivíduos são necessários, para comprovar a utilização inadequada destes fármacos na população estudada.

\section{Referências}

Axfors, C., Schmitt, A. M., Janiaud, P., van't Hooft, J., Abd-Elsalam, S., Abdo, E. F., \& Hemkens, L. G. (2021). Mortality outcomes with hydroxychloroquine and chloroquine in COVID-19 from an international collaborative meta-analysis of randomized trials. Nature communications, 12 (1), 1-13.

Beltrão, R. P. L., Mouta, A. A. N., Silva, N. S., Oliveira, J. E. N., Beltrão, I. T., Beltrão, C. M. F., \& da Silva, A. C. B. (2020). Perigo do movimento antivacina: análise epidemio-literária do movimento antivacinação no Brasil. Revista Eletrônica Acervo Saúde, 12 (6), e3088-e3088.

Boulware, D. R., Pullen, M. F., Bangdiwala, A. S., Pastick, K. A., Lofgren, S. M., Okafor, E. C., \& Hullsiek, K. H. (2020). A randomized trial of hydroxychloroquine as postexposure prophylaxis for Covid-19. New England Journal of Medicine, 383(6), 517-525.

Brasil, M. S. (2020a). Orientações do Ministério da Saúde para manuseio medicamentoso precoce de pacientes com diagnóstico da Covid-19. Ministério da Saúde.

Brasil, M. S. (2020b) Nota Informativa no 17/2020- SE/GAB/SE/MS. Orientações do Ministério da Saúde para manuseio medicamentoso precoce de pacientes com diagnóstico da Covid-19. http://antigo.saude.gov.br/images/pdf/2020/August/12/COVID-11ago2020-17h16.pdf.

Brasil. (2020b). Portaria MS/GM nº 188, de 3 de fevereiro de 2020. Declara Emergência em Saúde Pública de importância Nacional (ESPIN) em decorrência da Infecção Humana pelo novo Coronavírus (2019-nCoV). Diário Oficial da União.

Centers for Disease Control and Prevention (2020). Interim clinical guidance for management of patients with confirmed coronavirus disease (COVID-19) https://www.cdc.gov/coronavirus/2019-ncov/hcp/clinical-guidance-management-patients.html.

Chaccour, C., Hammann, F., Ramón-García, S., \& Rabinovich, N. R. (2020). Ivermectin and COVID-19: keeping rigor in times of urgency. The American journal of tropical medicine and hygiene, 102(6), 1156.

Gautret, P., Lagier, J. C., Parola, P., Meddeb, L., Mailhe, M., Doudier, B., \& Raoult, D. (2020). Hydroxychloroquine and azithromycin as a treatment of COVID-19: results of an open-label non-randomized clinical trial. International journal of antimicrobial agents, $56(1), 105949$.

Gostin, L. O. (2020). The great coronavirus pandemic of 2020 - 7 critical lessons. Jama, 324(18), 1816-1817.

Hongchao, P., Peto, R., Henao-Restrepo, A. M., Preziosi, M. P., Sathiyamoorthy, V., Karim, Q. A., \& Manuel, O. (2020). Repurposed antiviral drugs for COVID-19: interim WHO solidarity trial results (Preprint).

International Committee on Taxonomy of Viruses (2011). Coronaviridae-Positive Sense, R. N. A. Viruses-Positive Sense RNA Viruses https://talk.ictvonline.org/ictv-reports/ictv_9th_report/positive-sense-rna-viruses-2011/w/posrna_viruses/222/coronaviridae.

Lima, C. H. (2021). História psicossocial das emergências humanitárias: Uma sistematização da resposta brasileira ao impacto da COVID-19 na saúde da população. Research, Society and Development, 10(8), e30610817275-e30610817275.

López-Medina, E., López, P., Hurtado, I. C., Dávalos, D. M., Ramirez, O., Martínez, E., \& Caicedo, I. (2021). Effect of ivermectin on time to resolution of symptoms among adults with mild COVID-19: a randomized clinical trial. Jama, 325(14), 1426-1435.

Mitjà, O., Corbacho-Monné, M., Ubals, M., Alemany, A., Suñer, C., Tebé, C., \& Clotet, B. (2020). A cluster-randomized trial of hydroxychloroquine for prevention of Covid-19. New England Journal of Medicine.

RECOVERY Collaborative Group. (2020). Effect of hydroxychloroquine in hospitalized patients with Covid-19. New England Journal of Medicine, 383(21), $2030-2040$

Rocco, P. R., Silva, P. L., Cruz, F. F., Melo-Junior, M. A. C., Tierno, P. F., Moura, M. A., \& e Silva, J. R. L. (2021). Early use of nitazoxanide in mild Covid19 disease: randomised, placebo-controlled trial. European Respiratory Journal, 58(1).

Santos-Pinto, C. D. B., Miranda, E. S., \& Osorio-de-Castro, C. G. S. (2021). O "kit-covid” e o Programa Farmácia Popular do Brasil. Cadernos de Saúde Pública, 37. 
Research, Society and Development, v. 10, n. 12, e287101220486, 2021

(CC BY 4.0) | ISSN 2525-3409 | DOI: http://dx.doi.org/10.33448/rsd-v10i12.20486

Secretaria de Estado de Saúde do Mato Grosso (2020). Veja a lista com as dez principais fake news sobre o coronavírus. http://www.mt.gov.br/-/14066969veja-a-lista-com-as-dez-principais-fake-news-sobre-o-coronavirus.

Secretaria de Estado da Saúde do Pará - SESPA (2020). Coronavírus http://www.saude.pa.gov.br/coronavirus.

Secretaria de Estado da Saúde do Pará - SESPA (2021a). Coronavírus http:// http://www.saude.pa.gov.br/vacinometro/.

Secretaria de Estado da Saúde do Pará - SESPA (2021b). Plano Paraense de Vacinação - PPV/COVID-19. (4a ed.), http://www.saude.pa.gov.br/coronav irus/nota-tecnica-covid/.

Singh, B., Ryan, H., Kredo, T., Chaplin, M., \& Fletcher, T. (2020). Chloroquine or hydroxychloroquine for prevention and treatment of COVID-19. The Cochrane database of systematic reviews, 2020(4).

World Health Organization (2021). Coronavirus Disease (COVID-19) https://covid19.who.int/.

World Health Organization (2020). OMS declara emergência de saúde pública de importância internacional por surto de novo coronavírus. https://www.paho.org/bra/index.php?option=com_content\&view=article\&id=6100:oms-declara-emergencia-de-saude-publica-de-importancia-internacionalem-relacao-a-novo-coronavirus\&Itemid= 812 .

Yan, Y., Zou, Z., Sun, Y., Li, X., Xu, K. F., Wei, Y., \& Jiang, C. (2013). Anti-malaria drug chloroquine is highly effective in treating avian influenza A H5N1 virus infection in an animal model. Cell research, 23(2), 300-302.

Zhu, N., Zhang, D., Wang, W., Li, X., Yang, B., Song, J., \& Tan, W. (2020). A novel coronavirus from patients with pneumonia in China, 2019. New England journal of medicine. 